\title{
Correction to "Update on Gaseous Signaling Molecules Nitric Oxide and Hydrogen Sulfide: Strategies to Capture their Functional Activity for Human Therapeutics"
}

In the above article [Bryan NS and Lefer DJ (2019) Mol Pharmacol, 96(1): 109-114; DOI: https://doi.org/10.1124/mol.118.113910], the following funding information was omitted:

This work was supported by the National Institutes of Health [Grants 1R44 HL139195-01, 1R44 HL139161-01, 1R01 HL146098-01, and 1R44 HL136233-01 (to DJL)].

The HTML and PDF versions of the article have been updated to include this information. The authors regret this error and any inconvenience it may have caused. 